\title{
Laparoscopic Cholecystectomy in Situs Inversus Totalis Patient: Our Experience
}

\author{
Gulzar Ahmad Bhat* and Deepak Ghulliani \\ Department of General Surgery, Maulana Azad Medical College, India
}

*Corresponding author: Gulzar Ahmad Bhat, MBBS, MS, Registrar Department of General Surgery, Maulana Azad Medical College, New Delhi, India

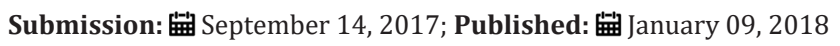

\begin{abstract}
Situs inversus is an autosomal recessive anomaly characterized by transposition of abdominal viscera, and when associated with a right sided heart (Dextrocardia) is referred to as situs inversus totalis. Mathew Baillie was first to described situs inversus totalis in early twentieth century. Complete situs inversus is a rare syndrome, of autosomal recessive inheritance, with an estimated prevalence of around $1 / 10000$ births. The frequency of cholelithiasis in patients with situs inversus is similar to that in the general population. However, the condition may present diagnostic difficulty. In the published literature, there have been only 40 reports of open cholecystectomy in pre-laparoscopic era and 21 reports of laparoscopic cholecystectomy in situs inversus patients till date. We present the case of a 21 year old male with situs inversus totalis with symptomatic cholelithiasis which was successfully treated with Laparoscopic cholecystectomy. The mirror image anatomy poses difficulty in orientation during laparoscopic cholecystectomy in patients with situs inversus. The surgeon needs to reorient visual images and surgical steps in an anatomical field that has undergone $180 \AA$ rotation. We conclude that though laparoscopic cholecystectomy in patients with situs inversus is technically more demanding although an experienced laparoscopic surgeon can perform it safely.
\end{abstract}

Keywords: Situs Inversus ; Cholelithiasis ; Laparoscopic Cholecystectomy.

\section{Introduction}

Situs inversus is an autosomal recessive anomaly with transposition of abdominal viscera, and if associated with a right sided heart (Dextrocardia) is referred to as situs inversus totalis [1]. Fabricius reported the first known case of situs inversus in humans in 1600 [2]. It was Mathew Baillie who first described situs inversus totalis in early twentieth century [3]. Transposed abdominal organs are a mirror image of the normal anatomy [4]. Situs inversus may be associated with a number of other conditions like Kartagener's syndrome and cardiac and respiratory anomalies. The frequency of cholelithiasis in patients with situs inversus is similar to that in the general population [5]. However, the condition may present diagnostic difficulty. Pain of biliary colic may be located in the epigastrium or in the left subcostal region and that of cholecystitis is radiates to the left infrascapular region.

\section{Case Presentation}

We present the case of a 21 year old male who presented to our OPD with complaint of pain left upper abdomen referred to left shoulder and radiated to inferior angle of left scapula for 4 months. General physical examination was normal. Apex beat was heard on right side of chest just outside mid clavicular line in 5th inter-costal space. On abdominal examination liver dullness couldn't be elicited on examination of right upper abdomen. Rest of clinical examination was essentially normal. Laboratory investigations were normal.
Chest radiograph showed cardiac shadow mainly on right side with normal bilateral lung fields (Figure 1). On ultrasonography (USG) abdomen liver, gall bladder (GB) and common bile duct (CBD) were seen as mirror image of normal with multiple calculi in the lumen of GB. Pancreas was grossly normal but on right side and spleen in right upper abdomen. Both kidneys were normal. A diagnosis of SYMPTOMATIC CHOLELITHIASIS WITH SITUS INVERSUS TOATLIS was made. Patient was taken up for laparoscopic cholecystectomy under general anesthesia after pre-anesthetic work-up.

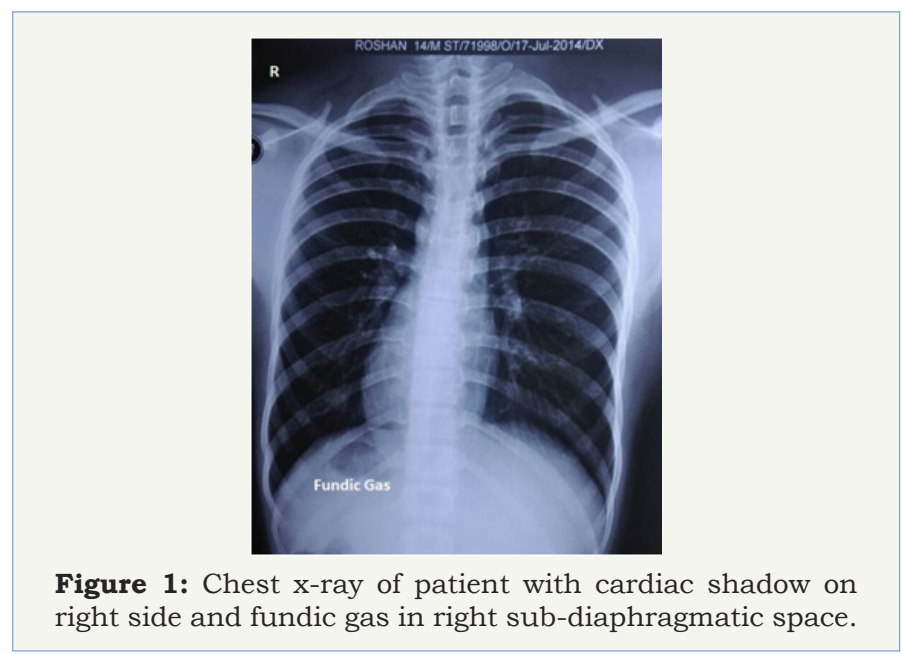




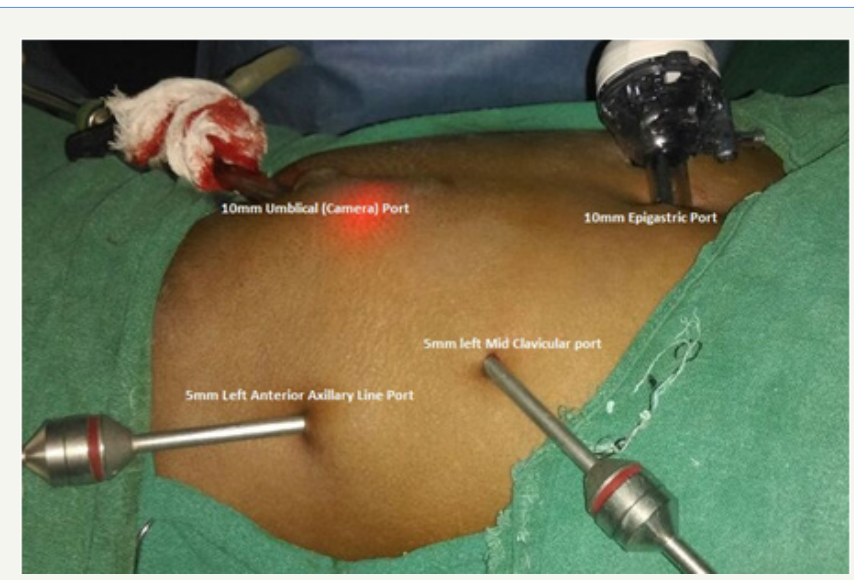

Figure 2: Image showing position of various ports for laparoscopic cholecystectomy.

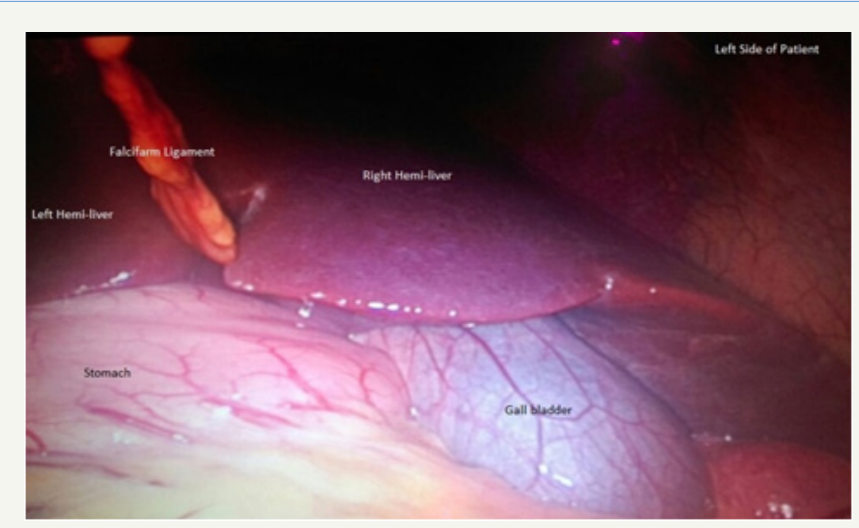

Figure 3: Image showing stomach, liver and falcifarm ligament with gall bladder on its left.

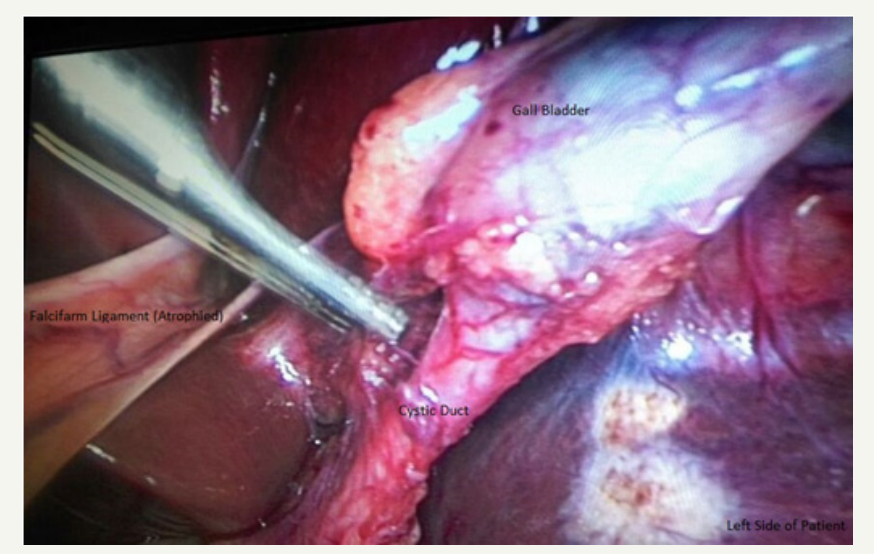

Figure 4: Image showing calot's triangle anatomy.

Patient was positioned in slight reverse Trendlenberg position with left up. Four port laparoscopic cholecystectomy was done with two $10 \mathrm{~mm}$ ports at umbilicus and epigastrium and two $5 \mathrm{~mm}$ ports in left midclavicular and left anterior axillary line in subcostal region (Figure 2). Pneumo-peritonium was created by open technique via umbilical port. Other ports were put under vision. Umbilical port was used as camera port. Calot's triangle anatomy was mirror image of normal. Dissection was done through midclavicular port and epigastric port was used to manipulate the gallbladder (Figure 2-4). The right handed surgeon faced some difficulty in orientation of structures and instruments. Total operative time was 50 minutes which was about 10 minutes more than the average time for routine laparoscopic cholecystectomy by us.

\section{Discussion}

In the published literature, there have been only 40 reports of open cholecystectomy in pre-laparoscopic era and 21 reports of laparoscopic cholecystectomy in situs inversus patients till date $[2,6,7]$. The mirror image anatomy poses difficulty in orientation during laparoscopic cholecystectomy in patients with situs inversus. Various laparoscopy ports need to be positioned at sites that are mirror image of those in the usual patient. The surgeon needs to reorient visual images and surgical steps in an anatomical field that has undergone $180 ®$ rotation. Right handed surgeon can use midclavicular port for dissection or can do dissection with left hand through epigastric port. Though laparoscopic cholecystectomy in patients with situs inversus is technically more demanding, an experienced laparoscopic surgeon can perform it safely. There have been no reports of conversion of laparoscopic cholecystectomy to open cholecystectomy in patients with situs inversus in literature.

\section{Conclusion}

We conclude that though laparoscopic cholecystectomy in patients with situs inversus is technically more demanding, an experienced laparoscopic surgeon can perform it safely.

\section{Objective}

The objective of sharing of our experience is to highlight the difficulties faced by the left handed surgeons during routine laparoscopic surgeries thus highlighting the importance of dexterity for a surgeon.

\section{References}

1. Applegate KE, Goske MJ, Pierce G, Murphy D. Situs revisited: Imaging of heterotaxy syndrome. Radiographics 1999; 19 (4): 837-52.

2. Takei HT, Maxwell JG, Clancy TV, Tinsley EA. Laparoscopic cholecystectomy in situs inversus totalis. J Laparoendosc Surg 1992; 2:171-6.

3. Harler DJ, O' Leary PW : Cardiac malpositions and abnormalities of atrial and visceral situs In: Emmanourlides GC, Riemenschnerder TA, Allen HD, Gutesell HP (eds). Heart Disease in Infants, Children, Adolescents. 5th ed. Vol 2. Williams \& Wilkins; 1995: 1307-36.

4. Higgins CB. Essentials of Cardiac Radiology and Imaging. Lippincot 1992; 283-331.

5. Banerjee JS, Vyas FL, Jesudason MR, Govil S and Muthusami JC. Laparoscopic cholecystectomy in a patient with situs inversus. Indian J Gastroenterol 2004;23:79-80.

6. McKay D, Blake G. Laparoscopic cholecystectomy in situs inversus totalis:a case report. BMC Surg 2005;5:5.

7. Oms LM, Badia JM. Laparoscopic cholecystectomy in situs inversus totalis- the importance of being left handed. Surg Endosc 2003;17:185961 